\title{
Clinical research on dendritic cell vaccines to prevent postoperative recurrence and metastasis of liver cancer
}

\author{
T.Y. Sun, W. Yan, C.M. Yang, L.F. Zhang, H.L. Tang, Y. Chen, H.X. Hu and X. Wei \\ General Surgery Department, Air Force General Hospital, Beijing, China \\ Corresponding author: X. Wei \\ E-mail: suntiye_ty@163.com \\ Genet. Mol. Res. 14 (4): 16222-16232 (2015) \\ Received June 28, 2015 \\ Accepted September 4, 2015 \\ Published December 8, 2015 \\ DOI http://dx.doi.org/10.4238/2015.December.8.12
}

\begin{abstract}
We aimed to evaluate dendritic cell (DC) tumor vaccines for preventing liver cancer recurrence and metastasis. DCs were induced from mononuclear cells in the peripheral blood with recombinant human granulocyte macrophage colony stimulating factor (rhGM-CSF) and recombinant human interleukin 4 (rhlL-4), followed by sensitization with lysis of autologous liver cancer cells. One hundred and sixty patients with hepatocellular carcinoma were randomly divided into two groups of 80 . One group was treated postoperatively with six cycles of the DC tumor vaccine. The other group was treated postoperatively with six cycles of FOLFOX 6, beginning 1 week after surgery. After treatment with DC tumor vaccines, the levels of CD3+, CD4+, and CD8+, the ratio of CD4+ to $\mathrm{CD} 8+\mathrm{DC}$, and the serum levels of IL-12 and IFN- $\gamma$ were significantly increased both in comparison to the pre-treatment levels $(P<0.001)$ and to the chemotherapy group $(P<0.001)$. After a postoperative follow-up of 18 months, the metastatic recurrence rate in the DC tumor vaccine group was significantly lower than that in the chemotherapy group (17.50 vs $48.75 \%$, $P<0.005)$, and the survival rate of the patients in the DC tumor vaccine group was higher than that of the chemotherapy treatment group (86.25 vs $52.50 \%, \mathrm{P}<0.005)$. Treatment with $\mathrm{DC}$ tumor vaccines was safe and
\end{abstract}


feasible. It can enhance the immunity of the patients, reduce the rates of metastasis and recurrence, and improve survival rates. This is a promising treatment for the prevention of postoperative recurrence in patients with liver cancer.

Key words: Liver; Dendritic cells; Immune treatment; Chemical treatment; Recurrence; Metastasis

\section{INTRODUCTION}

Liver cancer is currently the fifth most common malignancy worldwide. Although radical resection is the most effective treatment for primary liver cancer, $60-70 \%$ of patients relapse within 5 years, which leads to a high local recurrence rate (Banchereau et al., 2001). Therefore, a positive and effective method to prevent postoperative recurrence is needed, and this is an active area of research. In a recent study, dendritic cells (DCs) were discovered to be the most powerful antigenpresenting cells (APC) in the body's immune system, playing a key role in initiating and controlling the immune response (Yamanaka et al., 2005; Perambakam et al., 2006).

Our previous studies have shown that DCs can effectively induce resistance to gastric cancer (Sun et al., 2010a), and in recent years, a tumor vaccine based on DCs has been effective in the treatment of melanoma (Banchereau et al., 2001). However, the study of prevention of postoperative recurrence and metastasis in patients with hepatocellular carcinoma is still at the exploration stage.

Therefore, we conducted a randomized controlled trial comparing a DC tumor vaccine to chemotherapy. The outcomes of interest were recurrence rates, survival, and immune functions.

Therefore, this study recruited 160 patients with liver cancer who met the inclusion criteria and underwent curative resection at the Department of Hepatobiliary Surgery at our hospital from July 2008 to July 2011 . The cases were randomly divided into two groups, one to be treated with DC tumor vaccines and the other, the chemical treatment group, to be treated with chemotherapy.

Peripheral blood was extracted from the patients in the DC tumor vaccine group and then the DCs were induced from the peripheral blood mononuclear cells (PBMC) by stimulation with recombinant human granulocyte macrophage colony stimulating factor (rhGM-CSF), recombinant human interleukin (rhIL-4), and other activating cytokines. The DCs were primed by injecting autologous liver cancer cell lysis into the patient's body 1 week later. We also discuss the immune function of DC tumor vaccines sensitized by autologous liver cancer cell lysis to stimulate the body to resist liver cancer, a new technique for postoperative biological immunotherapy of liver cancer patients.

\section{MATERIAL AND METHODS}

\section{Clinical data}

We recruited 160 patients with liver cancer who underwent curative resection in the Department of Hepatobiliary Surgery at our hospital from July 2008 to July 2011 for our randomized controlled study. All of the patients had resected metastases or were without distant metastases, none had serious viscera insufficiency, and they were all diagnosed with hepatocellular carcinoma by histologic examination postoperatively. 
The patients were divided into two groups at random using a digital method. Eighty cases were assigned to the chemical treatment group, 56 men and 24 women, with an average age of $53.2 \pm 7.5$ years. After surgery, they underwent chemical treatment according to the standard chemotherapy regimen FOLFOX 6.

Eighty cases were assigned to the DC tumor vaccine treatment group, 56 men and 24 women with an average age of $57.3 \pm 7.1$ years. After surgery, these patients were given six cycles of DC tumor vaccine treatment.

The two groups were comparable in age, gender, alpha-fetoprotein (AFP) levels, tumor size, tumor differentiation degree, the degree of liver cirrhosis, and whether the cancer was infringing on the vascular system $(P>0.05)$.

This randomized controlled trial was approved by the medical Ethics Committee. All of the patients and their families signed the informed consent.

All patients were not receiving systemic chemotherapy. The inclusion criteria were: 1) a single tumor; 2) no preoperative blood transfusion; 3) the pathological diagnosis was hepatocellular carcinoma; 4) the preoperative examination found no distant metastases; 5) the cut edge of the tumor was larger than $1 \mathrm{~cm}$; 6) the Karnofsky score was greater than 70; 7) the patients were compliant with treatment and could be followed up easily. There were no other specific exclusion criteria.

\section{Main reagents and instruments}

AIM-V serum-free medium was provided by the Gibco company. Ficoll lymphocyte separation medium was provided by the Lymphoprep company. rhGM-CSF, rhIL-4, and tumor necrosis factor (TNF)- $\alpha$ were provided by the PeproTech company. Fluorescence-tagged (FITC) monoclonal antibodies, including cD3-Tc, IL-4-PE, and IFN- $\gamma$ were provided by the Caltag company; CD8-FITC and CD4-PE were provided by the BD Pharmingen company. The FACS Calibur flow cytometry (FCM) instrument was provided by the BD company.

\section{Separation of PBMC}

Patients were injected subcutaneously with $100 \mu \mathrm{g}$ recombinant rhGM-CSF every day for mobilization of monocytes. After 5 days, $100 \mathrm{~mL}$ fresh peripheral blood with anticoagulation was collected. PBMC were separated by Ficoll density gradient centrifugation.

\section{Preparation for freeze-thaw lysis of the liver tumor}

The tumor tissue was cut up and placed in liquid nitrogen for $10 \mathrm{~min}$, followed by a water bath at $37^{\circ} \mathrm{C}$. The freeze-thaw cycle was repeated three times. The tissue was then further broken open with an ultrasonic instrument. The released fluid was collected by centrifugation and filtering through a $0.22-\mu \mathrm{m}$ microporous membrane. The total protein content was measured by the Biuret method, and the protein concentration was adjusted to $1.0 \mathrm{~g} / \mathrm{L}$.

\section{Preparation of DC tumor vaccines}

The PBMC were collected and washed twice, and adjusted to $5 \times 10^{6}$ cells per milliliter. They were placed in a culture bottle at $37^{\circ} \mathrm{C}$ with $5 \% \mathrm{CO}_{2}$ for $2 \mathrm{~h}$. The non-adherent cells were 
washed out and the adherent cells were cultivated in RPMI 1640 media including rhGM-CSF (100 $\mu \mathrm{g} / \mathrm{L})$ and IL-4 (100 $\mu \mathrm{g} / \mathrm{L})$ for 1 day, after which the nutrient solution was replaced and cytokines were added. On the fifth day, the liver lysis antigens $(100 \mu \mathrm{g} / \mathrm{mL})$ and TNF- $\alpha(100 \mu \mathrm{g} / \mathrm{mL})$ were added. The tumor vaccines were collected on the eighth day.

\section{Chemotherapy treatment}

The chemical treatment group was given chemotherapy 1 week after surgery with the FOLFOX 6 chemotherapy regimen described below:

Oxaliplatin at $100 \mathrm{mg} / \mathrm{m}^{2}$ was added in $500 \mathrm{~mL}$ glucose at a ratio of $5 \%$, and it was administered by intravenous drip over $180 \mathrm{~min}$ on the first day.

Leucovorin calcium at $200 \mathrm{mg} / \mathrm{m}^{2}$ in $250 \mathrm{~mL}$ saline was also administered by intravenous drip over $120 \mathrm{~min}$ on the first day.

Fluorouracil at $400 \mathrm{mg} / \mathrm{m}^{2}$ was administered by intravenous injection, and then a dose of $2400 \mathrm{mg} / \mathrm{m}^{2}$ was administered by a trace vein pump over $46 \mathrm{~h}$.

Five milligrams of tropane SiQiong was injected $30 \mathrm{~min}$ before chemotherapy in order to prevent patients from having digestive tract reactions such as nausea and vomiting. The treatment was repeated every 2 weeks, and 4 weeks was counted as one cycle. The treatment was discontinued in cases of disease progression, intolerance, or toxicity. The longest treatment cycle was 6 weeks.

Before and after each cycle of chemotherapy, the urine was tested for blood, and liver and kidney function, fasting blood glucose, blood AFP, and electrocardiogram information was collected. The curative effect was evaluated after two courses of treatment and at the 18-month follow-up time.

\section{DC tumor vaccines}

In the DC tumor vaccine treatment group, DC tumor vaccine treatment was administered 1 week after surgery. The vaccine was injected intradermally in the upper limbs, alternating between sides, with a dose of $8.9 \times 10^{9}$ cells per injection. An injection was given once a month, and each month was counted as one cycle of treatment. Six cycles of treatment were given in total. After each injection, patients were observed for adverse reactions such as fever and lateonset hypersensitivity.

\section{Observation method}

\section{Detection of immunological indexes}

Before and after the treatment with DC tumor vaccines, the T lymphocyte subgroup such as CD3+, CD4+ and CD8+ was detected in the peripheral blood by FCM. Five milliliters of peripheral blood with heparin anticoagulant was mixed with $10 \mu \mathrm{L}$ of the corresponding fluorescence-labeled antibody, and then incubated at $4^{\circ} \mathrm{C}$ for $20 \mathrm{~min}$. Then, $2 \mathrm{~mL}$ of the pyrolysis liquid was added, and it was placed at room temperature protected from light for $10 \mathrm{~min}$. The supernatant was discarded after centrifuging and the cells were washed twice with PBS. After fixing in $1 \%$ paraformaldehyde, the 
labeled cells were detected by FCM and the data were analyzed with the Cell Quest software. The positive expression rate of the obtained sample was $1 \times 10^{4}$, and the ones tagged with fluorescence of the same type of $\operatorname{lgG}$ as the corresponding negative control.

The levels of IL-10, IL-12, and IFN- $y$ in the serum were detected by an enzyme-linked immunosorbent assay. The concentration of peripheral blood cells was adjusted to $5 \times 10^{4} / \mathrm{mL}$, which was placed in six wells. After $4 \mathrm{~h}$ of culturing, we tested the levels of IL-10, IL-12 and IFN-Y in the cell supernatant.

\section{Clinical observation and follow-up}

Clinical evaluation was performed 1 week before the start of treatment, the 3rd week during the treatment, and 3 weeks after the treatment. The evaluation included a comprehensive collection of medical history, physical examination, routine blood tests, liver and kidney function tests, carcinoembryonic antigen levels, AFP levels, a total abdominal ultrasound, and a chest $\mathrm{X}$-ray examination. When the patients were suspected of having a tumor recurrence or metastasis, abdominal computed tomography $(\mathrm{CT})$ or magnetic resonance imaging (MRI) and puncture biopsy were performed. Every 3 months after surgery, the patients had serum levels of AFP, IL-10, IL-12, IFN-y measured, and they underwent an abdominal ultrasound, CT or MRI, and a chest X-ray. All patients were followed up until January 2013 or to the date of death.

\section{Statistical analysis}

The SPSS 16.0 statistical software was used to conduct data analysis. Results are reported as means \pm standard deviation. Chi-squared tests and $t$-tests were applied for group comparisons. All statistics were two-sided, with the level of significance set at $\alpha=0.05$. Therefore, a $\mathrm{P}<0.05$ means that the difference was statistically significant, and a $\mathrm{P}<0.01$ means that the difference was even more significant.

\section{RESULTS}

\section{Clinical observations of the DC tumor vaccine application}

The average number of DC induced in $100 \mathrm{~mL}$ peripheral blood was $9.2 \times 10^{10}$. The number of DC obtained was measured by FCM using the mature expression phenotype of DC (CD1a, CD83, CD86, CD80, CD40, and HLA-DR), (Table 1 and Figure 1).

\begin{tabular}{|c|c|c|c|c|c|c|}
\hline & CD1a & CD83 & CD86 & CD80 & CD40 & HLA-DR \\
\hline \multicolumn{7}{|c|}{ DC tumor vaccine treatment group } \\
\hline Before treatment & $49.05 \pm 3.27$ & $45.11 \pm 3.10$ & $56.33 \pm 3.54$ & $54.15 \pm 2.76$ & $58.39 \pm 2.83$ & $59.45 \pm 3.61$ \\
\hline After treatment & $74.12 \pm 3.29$ & $77.27 \pm 3.15$ & $78.51 \pm 3.48$ & $79.19 \pm 2.81$ & $78.44 \pm 2.87$ & $79.42 \pm 3.57$ \\
\hline \multicolumn{7}{|l|}{ Control group } \\
\hline Before treatment & $49.13 \pm 2.22$ & $50.43 \pm 2.52$ & $53.34 \pm 3.56$ & $54.28 \pm 2.32$ & $53.56 \pm 2.47$ & $54.27 \pm 3.34$ \\
\hline After treatment & $38.21 \pm 2.33$ & $37.48 \pm 2.61$ & $38.55 \pm 3.49$ & $40.31 \pm 2.42$ & $40.14 \pm 2.51$ & $41.19 \pm 3.29$ \\
\hline
\end{tabular}




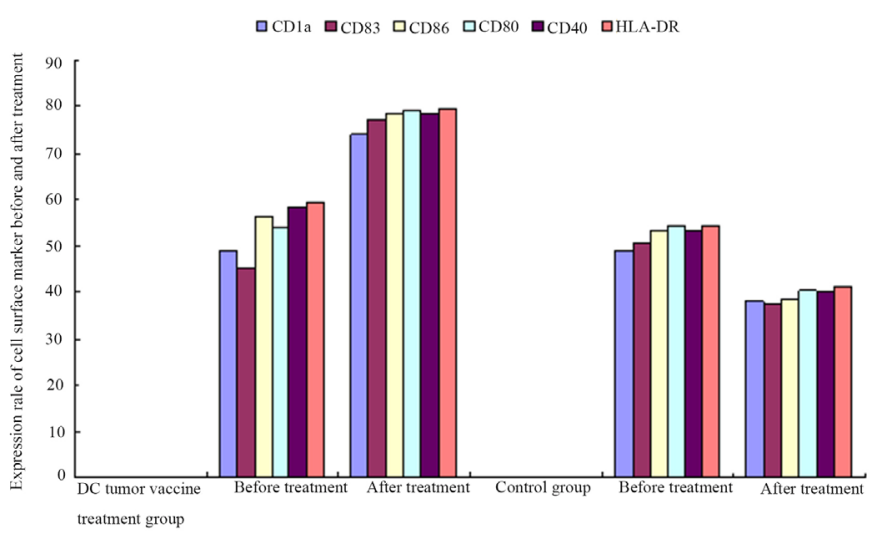

Figure 1. Expression rate of cell surface markers before and after treatment in the two groups (\%, means $\pm \mathrm{SD})$.

\section{Changes of T lymphocyte subsets before and after treatment in the two groups}

The levels of CD3+, CD4+, and CD4+/CD8+ in the peripheral blood of the DC tumor vaccine treatment group increased significantly relative to before treatment $(P<0.001)$, and the post-treatment levels were also significantly higher than that of the chemical group after treatment $(\mathrm{P}<0.001)$ (Table 2 and Figure 2).

Table 2. Changes of T lymphocyte subsets before and after treatment in the two groups (\% \pm SD).

\begin{tabular}{|c|c|c|c|c|}
\hline Groups & CD3+ & CD4+ & CD8+ & $\mathrm{CD} 4+/ \mathrm{CD} 8+$ \\
\hline \multicolumn{5}{|c|}{ DC tumor vaccine treatment group } \\
\hline Before treatment & $50.33 \pm 5.82$ & $39.56 \pm 7.29$ & $35.64 \pm 6.23$ & $0.97 \pm 0.42$ \\
\hline After treatment & $73.29 \pm 6.65^{*}$ & $67.62 \pm 8.14^{*}$ & $66.37 \pm 7.42^{*}$ & $1.68 \pm 0.59$ \\
\hline \multicolumn{5}{|l|}{ Control group } \\
\hline Before treatment & $52.14 \pm 4.37$ & $38.46 \pm 5.11$ & $33.55 \pm 4.72$ & $1.21 \pm 0.53$ \\
\hline After treatment & $46.17 \pm 5.22$ & $34.39 \pm 6.02$ & $31.23 \pm 5.52$ & $1.13 \pm 0.76$ \\
\hline
\end{tabular}

*Means compared with the DC tumor vaccine treatment group before treatment, $t=3.56,4.62,5.87$, $\mathrm{P}$ values $<0.001$, Compared with the control group after treatment, $t=3.27,4.83,5.72$, P values $<0.001$ to 0.01 .

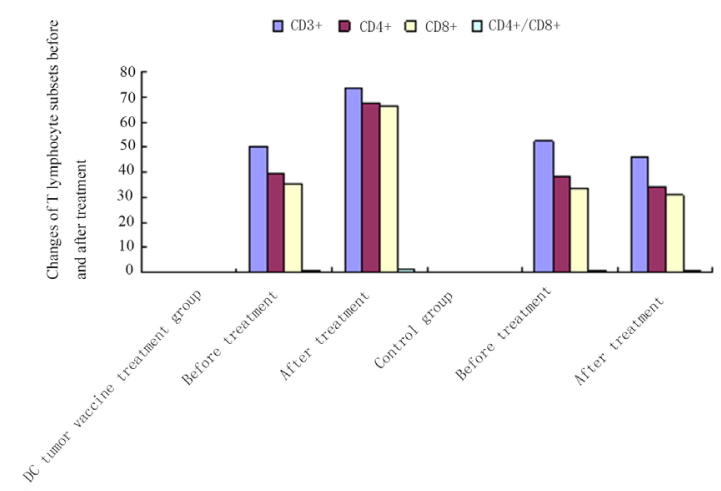

Figure 2. Changes of T lymphocyte subsets before and after treatment in the two groups (\%, means $\pm \mathrm{SD})$. 


\section{Changes of of IL-10, IL-12 and IFN- $-y$ levels in the serum in the two groups}

The concentration of IL-10 in the serum was $136.11 \pm 16.53 \mathrm{ng} / \mathrm{L}$ in the $\mathrm{DC}$ tumor vaccine treatment group after treatment, which was significantly lower than that before treatment $(375.45 \pm$ $17.34 \mathrm{ng} / \mathrm{L}, \mathrm{P}<0.01$ ). The concentration of IL- 2 and IFN-y in the serum of the DC tumor vaccine treatment group after treatment was $1083.43 \pm 54.67$ and $1079.25 \pm 54.74 \mathrm{ng} / \mathrm{L}$, respectively, which was significantly higher than that before treatment $(435.31 \pm 16.44$ and $425.13 \pm 17.05 \mathrm{ng} / \mathrm{L}$, respectively, $\mathrm{P}<0.001)$, and was also significantly higher than that in the chemical group after treatment $(516.41 \pm$ 18.58 and $531.38 \pm 19.08$ ng/L, respectively, $P<0.01$ ) (see Table 3 and Figure 3 ).

\begin{tabular}{|c|c|c|c|}
\hline Groups & IL-10 (ng/L) & IL-12 (ng/L) & IFN-y (ng/L) \\
\hline \multicolumn{4}{|c|}{$\mathrm{DC}$ tumor vaccines treatment group } \\
\hline Before treatment & $428.37 \pm 12.15$ & $526.38 \pm 13.27$ & $539.28 \pm 13.14$ \\
\hline After treatment & $129.28 \pm 12.22^{*}$ & $1265.41 \pm 62.34^{*}$ & $1312.34 \pm 63.38^{\prime}$ \\
\hline \multicolumn{4}{|l|}{ Control group } \\
\hline Before treatment & $394.26 \pm 12.39$ & $497.35 \pm 13.28$ & $542.59 \pm 13.82$ \\
\hline After treatment & $305.29 \pm 13.11$ & $601.39 \pm 25.72$ & $637.61 \pm 27.94$ \\
\hline
\end{tabular}

*Means $t=799.54,3.32,4.42$, P values $<0.001$ compared with DC tumor vaccine treatment group before treatment, $t=2.83,3.79,4.25$, $\mathrm{P}$ values $<0.001$ compared with the control group after treatment.

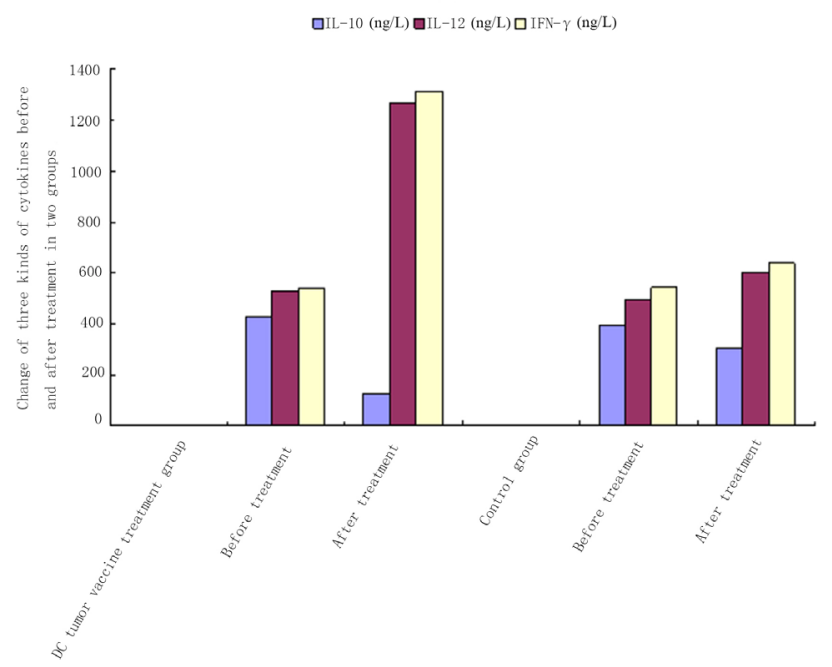

Figure 3. Changes of three kinds of cytokines before and after treatment in the two groups (\%, means \pm SD).

\section{Adverse reactions and safety evaluation}

After being inoculated with DC tumor vaccines, 11 patients experienced fever, with a temperature between $37.4^{\circ}$ to $38.3^{\circ} \mathrm{C}$; all patients returned to normal in 6 to $24 \mathrm{~h}$ without any special treatment. Twelve patients experienced a skin discoloration with a diameter of $1.5-2.6 \mathrm{~cm} 7$ days after the tumor vaccine injection, but all patients were able to tolerate it and the discoloration disappeared on its own without any special treatment after $72 \mathrm{~h}$. During the tumor vaccine 
immunization, no patients experienced symptoms such as nausea, vomiting, headache, or arthritis, and we did not observed any autoimmune reactions such as bronchial asthma and anaphylactic shock.

Some patients experienced different degrees of appetite improvement and pain relief, and their quality of life improved dramatically. Routine blood, liver, and kidney function, ultrasound of the abdomen and chest X-rays did not detect any significant abnormalities, and all patients tolerated the treatment very well.

\section{The follow-up situation}

The duration of follow-up was 18 months. In the DC tumor vaccine treatment group, five patients of the 80 experienced a local recurrence, six had a distant recurrence, and three had both local and distant recurrences, for an overall rate of recurrence of $17.50 \%$, which was significantly lower than that of the chemical treatment group $(48.75 \%, \mathrm{P}<0.005)$.

Eleven patients died in the DC tumor vaccine treatment group 11 to 18 months after the operation, for a survival rate of $86.25 \%$, which was significantly higher than that of the chemical treatment group $(52.50 \%, \mathrm{P}<0.005)$. In the chemical treatment group, 38 patients died 7 to 18 months after the operation, as shown in Table 4. Parts of transfer and recurrence were respectively in areas such as the lung and liver.

Table 4. Comparison of recurrence rate and survival rate in the two groups (\%).
\begin{tabular}{lccc}
\hline Groups & Number of cases & Rate of metastasis and recurrence & Rate of survival \\
\hline DC tumor vaccine treatment group & 80 & $17.50(14 / 80)^{*}$ & $86.25(11 / 80)^{\#}$ \\
Control group & 80 & $48.75(39 / 80)$ & $52.50(38 / 80)$ \\
\hline
\end{tabular}

Compared with control group, *means $\chi^{2}=10.16, \mathrm{P}<0.005$; " means $\chi^{2}=9.85, \mathrm{P}<0.005$.

Preoperative CT of nine cases in the chemical treatment group showed that there was a large block of liver cancer in the left and right hepatic lobes. The mean serum AFP of these patients was $8634.13 \pm 11.27 \mu \mathrm{g} / \mathrm{L}$, IL-10 was $385.36 \pm 13.53 \mathrm{ng} / \mathrm{L}$, IL-12 was $473.39 \pm 23.47 \mathrm{ng} / \mathrm{L}$ and the IFN-y was $514.57 \pm 13.28 \mathrm{ng} / \mathrm{L}$. Chemotherapy was conducted 1 week after eradicative resection of the liver cancer, and the AFP at that time was $897.16 \pm 12.45 \mu \mathrm{g} / \mathrm{L}$, IL-10 was $292.24 \pm 13.51$ $\mathrm{ng} / \mathrm{L}$, IL-12 was $593.26 \pm 25.63 \mathrm{ng} / \mathrm{L}$, and the IFN-y was $611.55 \pm 27.43 \mathrm{ng} / \mathrm{L}$. At the sixth month of follow-up, the serum AFP was $897.16 \pm 12.45 \mu \mathrm{g} / \mathrm{L}$, IL-10 was $292.24 \pm 13.51 \mathrm{ng} / \mathrm{L}$, IL-12 was 593.26 $\pm 25.63 \mathrm{ng} / \mathrm{L}$, and IFN-y was $611.55 \pm 27.43 \mathrm{ng} / \mathrm{L}$. CT showed that there were multiple low-density metastases in the right hepatic lobe after the resection of the left hepatic lobe, and there existed multiple low-density metastases in the left hepatic lobe after the resection of the right hepatic lobe.

After three treatments with the DC tumor vaccines the serum of these nine patients was reviewed and AFP was $112.04 \pm 11.23 \mu \mathrm{g} / \mathrm{L}$, IL-10 was $124.13 \pm 13.26 \mathrm{ng} / \mathrm{L}, \mathrm{IL}-12$ was $867.95 \pm$ $59.83 \mathrm{ng} / \mathrm{L}$, and the IFN- $\gamma$ was $963.75 \pm 27.66 \mathrm{ng} / \mathrm{L}$. CT showed that the low-density foci on the left or right hepatic lobe were narrower than before treatment.

\section{DISCUSSION}

Liver resection is the most effective treatment of primary liver cancer, but even after performed radical resection $60-70 \%$ of patients relapse within 5 years. At present, systemic chemotherapy for treating hepatocellular carcinoma postoperatively is largely ineffective, and 
there are undesirable effects on the immunity of patients. It is critical to explore new treatments to prevent and improve the problems caused by chemical treatments.

As a clinician, designing an effective biological immune therapy for prevention of postoperative recurrence was one of the problems to be solved at present. Early research suggests that DCs are the most powerful APC in the body. They can effectively absorb and process the tumor antigens and express high levels of major histocompatibility antigen complexes such as molecules of class I and II and various stimulus molecules such as CD80 and CD86, which play an important role in the tumor immune response (Mortara et al., 2007; Sun et al., 2010b). Some studies on the use of immunotherapy for treating solid tumors with DCs have been published (Lee et al., 2005). However, little research on enhancing the immune response for prevention of recurrence of postoperative of liver cancer has been performed.

This study induced DCs from the peripheral blood of patients with liver cancer, which were then activated by cytokines such as rhGM-CSF and rhlL-4. They were then induced to mature by TNF- $\alpha$ and sensitized by the cells of the autologous liver tumor. The DC tumor vaccines were injected into patients' bodies to explore their clinical effect on inducing immunity in patients with liver cancer and to assess their ability to prevent the recurrence and metastasis of postoperative liver cancer.

T-cell immunity is the core of the tumor immune response. Subsets of T lymphocytes often appear disordered in the peripheral blood of patients with tumors. CD4+ T cells can secrete cytokines to regulate antitumor immunity, and CD8+ T cells can secrete inhibitory factors that play a role of specific immune inhibition. Therefore, the ratio of CD4+ cells to CD8+ cells can reflect the body's immune function (Ruggieri et al., 2010). CD4+ T cells come in two different types, Th1 and Th2. The Th1 cells mainly mediate cell immune responses, by inducing T cell differentiation to Th1, which play an important role in antitumor immunity. Th2 cells mainly mediate humoral immunity, and induce antibodies to be generated. CD8+ T cells also come in two subtypes, Tc1 and Tc2. Tc1 cells secrete IFN- $y$, which has significant cytotoxic activity. Tc2 cells secrete IL-4, which is almost without cytotoxic activity. Tc1 cells are the main effectors of antitumor immune cells, which require IL-2 and IFN-y secreted by Th1 cells for activation.

After DC tumor vaccines were injected, Tc1, Tc2 and the ratio of CD4+ to CD8+ increased significantly, but there was no effect of DC tumor vaccines to induce the differentiation of Th1 in the body. This study examined the changes of the subtypes of CD3+, CD4+ and CD8+ in peripheral blood. The results showed that after administering the DC tumor vaccines, the number of CD3+, CD4+, and CD8+, and the ratio of CD4+ to CD8+ in peripheral blood were significantly increased. This suggests that this simple and easy method of preparing the DCs, which does not need a specific tumor antigen, can stimulate the body to produce a T lymphocyte immune response. If a more specific antigen had been used it may have induced Th1 to differentiate, which would be beneficial to further stimulate the body's anti-tumor immune response, and significantly improve the T lymphocytes' ability to recognize the tumor cells and induce a specific immune response to the liver cancer. As a result, it would kill the residual cancer cells and inhibit the metastatic recurrence of liver cancer.

DCs, as important APC cells, are becoming more important in the biological therapy of tumors (Lee et al., 2005). However, the number of DC in the tumor is limited, and the tumor can make DC dysfunction, apoptosis and absence of signaling molecule in an immune response, resulting in DC immunocompromised (Mailliard et al., 2000).

This study induced DCs from the mononuclear cells in the peripheral blood of the patients with rhGM-CSF and rhIL-4, and TNF- $\alpha$ was used to induce them to mature, after which lysis of autologous liver cancer cells was used to sensitize them. After injection into the patient, the DCs induce specific $\mathrm{T}$ lymphocyte immune cells to kill tumor cells. This method was simple, and it 
was not necessary to know the type(s) of tumor antigen, avoiding the difficulties of identification of tumor-specific antigens. This method also stimulated the $\mathrm{T}$ lymphocyte immune response to multiple tumor-associated antigens, which can reduce the possibility of tumor escape, as suggested by research conducted by Hoffmann et al. (2000).

At the same time, the diversity of antigen content generated by tumor cracking may lead to the induction of an autoimmune disease. However, our study found that the patients had no serious adverse reactions and had no occurrence of autoimmune disease through clinical observation and 18 months of follow-up. All patients tolerated the treatment well, and had significant improvements in quality of life. There were only 14 cases of recurrence and metastasis in the DC tumor vaccine group, with a recurrence rate of $17.50 \%$, and the survival rate was $86.25 \%$ through the eighteenth month of postoperative follow-up. In comparison, there were 39 cases of recurrence and metastasis in the chemical treatment group, and 38 patients died during the period from the seventh to the eighteenth month of postoperative follow-up. The survival rate was only $52.50 \%$, much lower than in the DC vaccine group, and the metastatic recurrence rate was also significantly lower in the DC tumor vaccines group.

There were 12 cases of delayed hypersensitivity among the 80 patients in the DC tumor vaccines group. Delayed hypersensitivity is a kind of immune response mediated by $\mathrm{T}$ cells and has been suggested to be a reliability index of the immune response, which showed that DC tumor vaccines had stimulated the immune responses in patients with liver cancer.

Recurrence of liver cancer is thought to be of two kinds. One is the intrahepatic spread of primary tumors. Disseminated lesions continue to grow, and tumor cells can be found upon clinical examination of the liver after resection of the primary tumor. Other is a change on the basis of chronic liver tumor recurrence. In our study, nine cases of recurrence due to intrahepatic metastasis of liver cancer sixth months after surgery in the chemical treatment group were treated with DC tumor vaccines. After three treatment cycles, the recurrent lesions were found to be significantly narrowed, which suggested that DC tumor vaccines are suitable for controlling trace residual cancer cells after curative surgery, which can improve the patient's immune function and prevent recurrences.

The DC tumor vaccine therapy is a good choice for patients with metastatic recurrence. The injection of DC tumor vaccines was only continued for 6 months in this study, which lead to an only limited ability to inhibit recurrence, but there is the possibility of only a limited effect on tumor recurrence, as suggested by the research of Takayama et al. (2000). Our results did not show that the survival rate was significantly improved overall, but the encouraging results have opened up a new approach to liver cancer immunotherapy. The related mechanisms need to be studied more in-depth in future studies.

Immunology indicators that estimate the prognosis of liver cancer include IL-10, IL-12, and IFN-y. IL-10 was significantly higher in the serum of the patients with liver cancer, but IL-12 and IFN-y were obviously lower. Single- and multiple-factor analysis has shown that the prognosis of patients with liver cancer with high levels of IL-10 and low levels of IL-12 and IFN-Y is poor (Chau et al., 2000; Wigginton et al., 2001; Parasole et al., 2001; Zhang and Wang, 2006; Airoldi et al., 2009). The results of our study show that treatment with DC tumor vaccines can result in a significant reduction in serum levels of IL-10, and increase IL-12 and IFN- $\gamma$, improving the body's anti-tumor immune response and inhibiting the recurrence and metastasis of liver cancer postoperatively.

In conclusion, this study found that treating patients with liver cancer postoperatively with DC tumor vaccines can delay the metastasis and recurrence of liver cancer. However, the curative effect on long-term follow-up is unknown. In addition, overall survival was not found to be obviously improved, for reasons that are not yet clear. We will continue to research this treatment with more cases and a longer period of DC tumor vaccine treatment, or combined with chemotherapy or 
targeted therapy in hopes that the effect of the overall treatment will improve the outcomes of patients with liver cancer. The anti-tumor immune liver cancer response, stimulated by DC tumor vaccines, is a promising new biological treatment for liver cancer.

\section{REFERENCES}

Airoldi ID, Carlo E, Cocco C, Caci E, et al. (2009). IL-12 can target human lung adenocarcinoma cells and normal bronchial epithelial cells surrounding tumor lesions. PLoS One 4: e6119.

Banchereau J, Palucka AK, Dhodapkar M, Burkeholder S, et al. (2001). Immune and clinical responses in patients with metastatic melanoma to CD34(+) progenitor-derived dendritic cell vaccine. Cancer Res. 61: 6451-6458.

Chau GY, Wu CW, Lui WY, Chang TJ, et al. (2000). Serum interleukin-10 but not interleukin-6 is related to clinical outcome in patients with resectable hepatocellular carcinoma. Ann. Surg. 231: 552-558.

Hoffmann TK, Meidenbauer N, Dworacki G, Kanaya H, et al. (2000). Generation of tumor-specific T-lymphocytes by crosspriming with human dendritic cells ingesting apoptotic tumor cells. Cancer Res. 60: 354 2-3549.

Lee WC, Wang HC, Huang PF, Lia CR, et al. (2005). Vaccination of advanced hepatocellular carcinoma patients with tumor 1ysate-pulsed dendritic cells. J. Immunother 28: 496-506.

Mailliard RB, Dallal RM, Son YI and Lotze MT (2000). Dendritic cells promote T-cell survival or death depending upon their maturation state and presentation of antigen. Immunol. Invest. 29:177-I85.

Mortara L, Giuliani L, De Lerma Barbaro A, Accolla RS, et al. (2007). Experimental therapeutic approaches to adenocarcinoma: the potential of tumor cells engineered to express MHC class II molecules combined with naked DNA interleukin-12 gene transfer. Surg Oncol 16: S33-S36.

Parasole R, Izzo F, Perrone F, Pignata S, et al. (2001). Prognostic value of serum biological markers in patients with hepatocellular carcinoma. Clin. Cancer Res. 7: 3504-3509.

Perambakam S, Hallmeyer S, Reddy S, Mahmud N, et al. (2006). Induction of specific T cell immunity in patients with prostate cancer by vaccination with PSA146-154 peptide. Cancer Immunol. Immunother 55: 1033.

Ruggieri A, Barbati C, and Momi W (2010). Cellular and molecular mechanisms involved in hepatocellular carcinoma gender disparity. Int. J. Cancer 127: 499.

Sun TY, Ye Wei, Liu QD, et al. (2010a). The preliminary study of the inducing culture of dendritic cells and the killing effect of the gastric cancer cell lines MKN45. J. Int. Onc. 37: 468-473.

Sun TY, Yan W, Liu QD, Zhou NX, et al. (2010b). The research on the killing effect of the dendritic cells in human peripheral blood stimulated by the CpG ODN1826 on gastric cancer cell. Chin. J. Clin. Phys. 4: 13-17.

Takayama T, Sekine T, Makuuchi M, Yamasaki S, et al. (2000). Adoptive immunotherapy to lower postsurgical recurrence rates of hepatocellular carcinoma: A randomised trial. Lancet 356: 802-807.

Wigginton JM, Gruys E, Geiselhart L, Subleski J, et al. (2001). TFN-gamma and Fas/FasL are required for the antitumor and antiangiogenic effects of IL-12/pulse IL-2 therapy. J. Clin. Invest. 108: 51-62.

Yamanaka R, Honma J, Tsuchiyayan, Yajima N, et al. (2005). Tumor lysate and IL-18 loaded dendritic cells elicits Th1 response, tumor specific CD8+cytotoxic T cells in patients with malignant glioma. J. Nero Oncol. 72: 107-113.

Zhang LJ and Wang XZ (2006). Interleukin-10 and chronic liver disease. World J. Gast. 12: 1681-1685. 